\title{
THE TRANSFER FUNCTION OF GENERAL TWO TERMINAL-PAIR RC NETWORKS*
}

\author{
BY \\ AARON FIALKOW \\ (Polytechnic Institute of Brooklyn) \\ AND \\ IRVING GERST \\ (Control Instrument Co., Brooklyn, N. Y.)
}

1. Introduction. Recent years have seen the increased use in many fields of passive electrical two terminal-pair networks lacking inductive elements. In particular, those networks having a common ground have found widespread employment in servomechanism design. In these applications, the network function of prime interest is generally the transfer function $A(p)$ defined as the ratio of steady state output voltage to input voltage in the domain of the complex frequency variable $p$.

Our discussion of the transfer function in the sequel will be given in terms of RCnetworks. However, it is known that by means of simple transformations the results in the RC-case can be made applicable to networks containing any two kinds of elements only. We will indicate these transformations later for LC-networks, important in classical filter theory, and for RL-networks. The actual statement of our results for these two cases is left as an exercise for the reader.

Despite its importance, there have been few investigations of any generality concerned with the transfer function. Being given a class of networks, the basic questions with regard to the transfer function are: (1) by what properties may one characterize the transfer functions which arise from this class of networks; (2) given a transfer function having these properties, how does one obtain corresponding networks of the class. If, as we may, we write

$$
A(p)=K N / D=K\left(p^{n}+a_{1} p^{n-1}+\cdots+a_{n}\right) /\left(p^{m}+b_{1} p^{m-1}+\cdots+b_{m}\right)
$$

where $N$ and $D$ have no common factors, we require a complete characterization of $K, N$ and $D$ for the class of networks under discussion as well as a synthesis procedure. It is important to note that since we are interested in what can be obtained from the networks alone without amplifiers, transformers or similar devices, the description of the multiplicative constant $K$ is just as essential as that of $N$ or $D$.

With this view of the problem in mind, we find that the bulk of the literature on RC transfer functions, besides concerning itself with quite special networks, only partially treats the questions here raised. Thus, generally, the constant $K$ is ignored, and incomplete conditions on $N$ and $D$ are given. The complete problem as stated here has thus far been investigated in the case of rather specialized classes of networks. Bower and Ordung [2] have considered the transfer function of the symmetric lattice using a geometric method while we [4] have treated this network analytically as well as the $L$ and general ladder networks.

In the present paper, we do not restrict ourselves to networks of any special internal

*Presented to the American Mathematical Society, February 24, 1951. (Cf. Bulletin of the American Mathematical Society, Vol. 57 (1951) pp. 182-3.) 
structure but treat the general grounded two terminal-pair (3 external terminals) and also the general two terminal-pair (4 external terminals) RC networks (abbreviated 3 T.N., 4 T.N., respectively.) An informal description of our results follows. For more precise statements, see Theorems 1 and 2. It is well known that the transfer function of a 3 T.N. or 4 T.N. is a rational function with real coefficients, regular at infinity, and having negative real, simple poles. In addition to these properties, we find that for the 3 T.N., the zeros of $N$ may not be positive real. For given $N$ and $D, K$ must be a number in the interval $0<K<K_{0}$, where $K_{0}$ is a constant depending upon $N$ and $D$ which is explicitly determined. Conversely, a 3 T.N. may be constructed which realizes any transfer function $A(p)$ whose $K, N, D$ satisfy the preceding conditions. In this connection, Guillemin [5] had previously shown that if $a_{i} \geq 0$ in $N$, then a 3 T.N. exists realizing the transfer function $A(p)$ for some unspecified $K$. In the case of the 4 T.N., we find that there is no restriction on the zeros of $N$. It is necessary and sufficient that $K$ lie in an interval $-K_{0}<K<K_{0}$, where again $K_{0}$ is given explicitly in terms of $N$ and $D$.

With regard to the above results, we remark that they do not imply the corresponding complete theorems for any structural sub-classes of 3 T.N. or 4 T.N. such as were discussed in [2] and [4]. For the transfer functions associated with a sub-class have further distinctive properties which are peculiar to the particular internal network structure of that sub-class and which permit synthesis in that structure.

Finally, we note that the results of the paper may be modified to take account of any resistive load or source.

2. The grounded two terminal-pair network. Theorem 1. The necessary and sufficient conditions that a real rational function $A(p)$ given by (1.1) be the transfer function of an $R C-3 T . N$. are:

(i) The zeros of $D$ are distinct negative numbers.

(ii) The zeros of $N$ may not be positive real but are otherwise arbitrary.

(iii) $m \geq n$.

(iv) The number $K$ satisfies the inequalities $0<K<K_{0}$, where $K_{0}$ is the least of the three quantities* $K_{d}, b_{m} / a_{n}, 1$ if $m=n$ and of the first two quantities if $m>n$. If $K_{0} \neq K_{d}$ then $K$ may equal $K_{0}$. Here $K_{d}$ is the least positive value of $\kappa$ (if it exists) for which the equation $D-{ }_{\kappa} N=0$ has a positive multiple root.

Proof: (a) Necessity. As stated in the introduction, conditions (i) and (iii) are well known in the case of a 4 T.N. To establish these conditions it is sufficient to write the transfer function (1.1) in the equivalent form

$$
A(p)=Y_{12} / Y_{22}
$$

where $Y_{12}$ and $Y_{22}$ are the short circuit transfer and driving point admittances, respectively, of the 4 T.N. and then apply Cauer's results on RC admittances and Brune's residue conditions. (Cf. [6], pp. 134-136, 211-212, 216-218). Since a 3 T.N. may be considered as a 4 T.N. with one input and one output terminal joined together (i) and (iii) also apply to a 3 T.N.

*If $a_{n}=0$, omit $b_{m} / a_{n}$. It may be shown that in any case at least one of the three quantities $K_{d}$, $b_{m} / a_{n}, 1$ actually appears as an upper bound for $K$. 
For the proof that the remaining conditions are necessary, the 3 T.N. shown in Fig. 1 is considered upon a nodal basis. Here the ground terminal is taken as node 0 , the other input and output terminals as nodes 1 and 2 respectively. For our purpose the remaining nodes are identified so that each branch is an $R$ and a $C$ in parallel. Hence

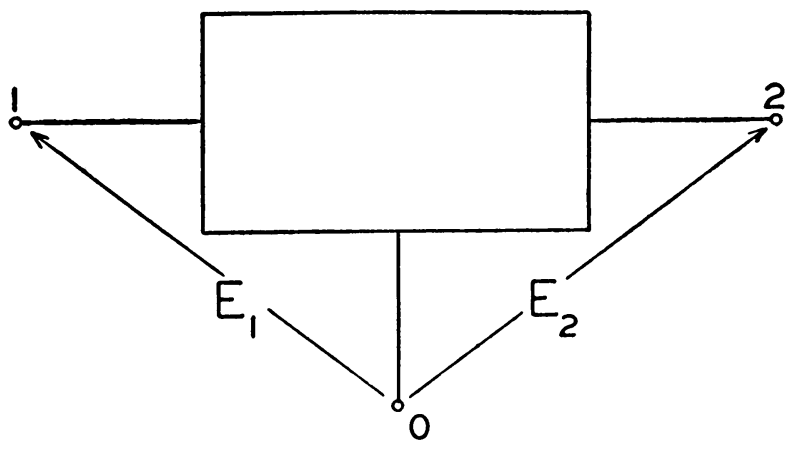

Fir. 1.

the admittance $y_{i j}(i \neq j)$ of the branch between nodes $i$ and $j$ is of the form $a p+b$, $a \geq 0, b \geq 0$. Of course $y_{i i}=y_{i i}$. Write

$$
y_{i i}=\sum_{\substack{i=0 \\ i \neq j}}^{t} y_{i j} \quad(i=1,2, \cdots, t),
$$

where $t+1$ is the total number of nodes. Then $y_{i i}$ is also linear in $p$ with non-negative coefficients. Let $I$ be the current impressed on node 1 by the driving source and denote by $E_{i}$ the voltage from node 0 to node $i$. As is well known [1], the equations of the nodal system are

$$
\begin{aligned}
& I=y_{11} E_{1}-y_{12} E_{2}-\cdots-y_{1 t} E_{t} \\
& 0=-y_{21} E_{1}+y_{22} E_{2}-\cdots-y_{2 \iota} E_{t} \\
& 0=-y_{t 1} E_{1}-y_{t 2} E_{2}-\cdots+y_{t} E_{t} .
\end{aligned}
$$

Since by definition $A(p)=E_{2} / E_{1}$, it follows from these equations that

$$
A(p)=\Delta_{1} / \Delta_{2},
$$

where

$$
\Delta_{1}=\left|\begin{array}{cccr}
y_{21} & y_{23} & \ldots & y_{2 t} \\
-y_{31} & y_{33} & \ldots & -y_{3 t} \\
\ldots \ldots \ldots \ldots \ldots \ldots \ldots & \ldots \ldots
\end{array}\right|=c_{0} p^{s}+c_{1} p^{s-1}+\cdots+c_{s},
$$




$$
\Delta_{2}=\left|\begin{array}{cccr}
y_{22} & -y_{23} & \cdots & -y_{2 t} \\
-y_{32} & y_{33} & \ldots & -y_{3 t} \\
\ldots \ldots & \ldots & \ldots & \ldots
\end{array}\right|=d_{0} p^{s}+d_{1} p^{s-1}+\cdots+d_{s}, d_{0} \neq 0 .
$$

Here $\Delta_{1}$ and $\Delta_{2}$ may have common factors and by (iii) the degree of $\Delta_{1}$ may actually be less than $s$.

It may be shown that*

$$
0 \leq c_{i} \leq d_{i} \quad(j=0,1, \cdots, s) .
$$

(In order not to interrupt the train of the argument at this point we defer the proof to Appendix A). It follows that none of the zeros of $\Delta_{1}$ and hence of $A(p)$ can be positive real. This establishes (ii). Also since $c_{i} \geq 0, d_{0}>0$, it follows that in the reduced form of the transfer function (1.1), $K>0$. Then by (ii), $a_{n} \geq 0$ in (1.1). (But note that some $a_{i}(i \neq n)$ may be negative).

In order to establish the necessity of (iv) let us suppose the roles of nodes 0 and 1 in Figure 1 are interchanged. This new 3 T.N. whose ground terminal is node 1 and whose other input and output terminals are nodes 0 and 2 respectively, while all other nodes are left unchanged, is termed the complementary network (of the original network) and its transfer function $A^{\prime}(p)$ is called the complementary transfer function. Then for this network under the same impressed current $I$ as before, the input voltage is $-E_{1}$ while the output voltage is $E_{2}-E_{1}$. Hence

$$
A^{\prime}(p)=\left(E_{2}-E_{1}\right) /-E_{1}=1-A(p) .
$$

Using (1.1), we find

$$
A^{\prime}(p)=(D-K N) / D,
$$

where it is clear that numerator and denominator are relatively prime. Hence the reduced form for $A^{\prime}(p)$ corresponding to (1.1) is

$$
A^{\prime}(p)=K^{\prime} \frac{p^{a}+a_{1}^{\prime} p^{a-1}+\cdots+a_{a}^{\prime}}{p^{m}+b_{1} p^{m-1}+\cdots+b_{m}},
$$

where, as shown above, $K^{\prime}>0, a_{a}^{\prime} \geq 0$. Comparison of the two expressions for $A^{\prime}(p)$ given by (2.7) and (2.8) shows that these last inequalities require $K \leq 1$ if $m=n$, $K \leq b_{m} / a_{n}$ if $a_{n} \neq 0$ respectively.

The remaining inequality on $K$ in (iv) is obtained by first showing that the transfer function of a 3 T.N. has what may be called the "interval property" with respect to its multiplicative constant $K$. That is, if $A(p)$ as given by (1.1) is the transfer function of a 3 T.N. then

$$
A^{*}(p)=K^{*} N / D, \quad 0<K^{*}<K,
$$

is also the transfer function of a 3 T.N.

To prove this, suppose $A(p)$ arises from the network of Figure 1. We may write

${ }^{*}$ That $d_{j} \geq 0$ is of course well known if either the conductance or capacitance matrix of the $y_{i j}$ occurring in $\Delta_{2}$ is positive definite. 
$A(p)$ as in (2.1). Now modify the 3 T.N. by introducing a new branch joining nodes 0 and 2 whose admittance is $\left(K-K^{*}\right) Y_{22} / K^{*}$. Then the modified 3 T.N. will have as short circuit driving point and transfer admittances $Y_{22}^{*}=Y_{22}+\left(K-K^{*}\right) Y_{22} / K^{*}$ and $Y_{12}^{*}=Y_{12}$ respectively; so that the corresponding transfer function is given by (2.9).

Now suppose $A(p)$ in (1.1) is the transfer function of a 3 T.N. and let $K_{d}$ be defined* as in Theorem 1, (iv). Then $K<K_{d}$. For by what has just been proved, if $K \geq K_{d}$, $A^{*}(p)=K_{d} N / D$ is also a 3 T.N. transfer function. But then the complementary transfer function $\left(D-K_{d} N\right) / D$ would have a positive real zero, in contradiction to (ii).

At first glance the bounds given for $K$ in Theorem 1, (iv) seem quite unrelated. However, one may alternatively characterize $K_{0}$ in (iv) as the greatest lower bound of all positive $\kappa$ for which the equation $D-{ }^{2} N=0$ has a positive real root. It may then be shown that this implies the statement as given in (iv).

(b) Sufficiency. Suppose now that $A(p)$ satisfies the conditions of Theorem 1 . We shall construct a 3 T.N. whose transfer function is $A(p)$. For this purpose, we first write $A(p)$ in a special form suggested by the fact that, before algebraic simplification, every transfer function has the form (2.3) with $d_{i} \geq c_{i} \geq 0$. We define this special form, called an $R$-function (realizable function), as follows: A real rational function

$R(p)=\left(g_{0} p^{l}+g_{1} p^{l-1}+\cdots+g_{l}\right) /\left(h_{0} p^{l}+h_{1} p^{l-1}+\cdots+h_{l}\right), \quad h_{0} \neq 0$,

is said to be an $R$-function if the zeros of its denominator are negative real and distinct, and if $0 \leq g_{i} \leq h_{i}(i=0,1, \cdots, l)$. We call $l$ the degree of the $R$-function. The expression of a rational function as an $R$-function is not unique.

It is shown in Appendix B that a function $A(p)$ satisfying the conditions of Theorem 1 may be written as an $R$-function. In the sequel, we give an algorithm for realizing every $R$-function as the transfer function of a 3 T.N. Our method consists of an induction on the degree $l$ of the $R$-function.

The case $l=0$ is trivial. If $l=1$, i.e., $R(p)=\left(g_{0} p+g_{1}\right) /\left(h_{0} p+h_{1}\right)$ with $h_{0} h_{1} \neq 0$, $0 \leq g_{0} \leq h_{0}, 0 \leq g_{1} \leq h_{1}$, then $R(p)$ is realized by an $L$-network whose series and shunt arm admittances are $Y_{a}=g_{0} p+g_{1}$ and $Y_{b}=\left(h_{0}-g_{0}\right) p+\left(h_{1}-g_{1}\right)$ respectively. Note that the $Y_{22}$ of this network is $h_{0} p+h_{1}$.

Now suppose that all $R$-functions of degree less than $l \geq 2$ are realizable as transfer functions of 3 T.N. Also assume that for $R(p)=U / V$ of degree $f<l, U$ and $V$ as in (2.10), the $Y_{22}$ of the corresponding network is of the form $V / S$ where $S$ is a polynomial of degree $f-1$. We will show that $(2.10)$ is realizable as the transfer function of a 3 T.N. such that its $Y_{22}$ is of the form $\sum_{i=0}^{l} h_{i} p^{l-i} / T$ where $T$ is a polynomial of degree $l-1$.

Let the negatives of the zeros of the denominator in (2.10) be $\gamma_{1}<\gamma_{2}<\cdots<\gamma_{l}$. Choose any $\sigma_{i}(i=1,2, \cdots, l-1)$ such that

$$
0<\gamma_{1}<\sigma_{1}<\gamma_{2}<\sigma_{2}<\cdots<\sigma_{l-1}<\gamma_{l} .
$$

Then the function $Z=h_{0} \prod_{i=1}^{l}\left(p+\gamma_{i}\right) / p \prod_{i=1}^{l-1}\left(p+\sigma_{i}\right)$ is an RC-impedance who canonical expansion is

$$
Z=h_{0}+A_{0} / p+\sum_{i=1}^{l-1} A_{i} /\left(p+\sigma_{i}\right), \quad A_{i}>0 \quad(i=0,1, \cdots, l-1) .
$$

*It follows that $K_{d}$ is one of the roots of the equation in $\kappa$ obtained by equating the discriminant of $D-\kappa N=0$ to zero. For the expression of the discriminant of an algebraic equation in terms of its coefficients see [3]. 
In (2.11) the terms of the right member with $h_{0}$ omitted form another RC-impedance which we write as $c \prod_{i=1}^{l-1}\left(p+\xi_{i}\right) / p \prod_{i=1}^{l-1}\left(p+\sigma_{i}\right), c>0$. We have

$$
0<\xi_{1}<\sigma_{1}<\xi_{2}<\sigma_{2}<\cdots<\xi_{l-1}<\sigma_{l-1} .
$$

From (2.11) it now follows that

$$
h_{0} \prod_{i=1}^{l}\left(p+\gamma_{i}\right)=h_{0} p \prod_{i=1}^{l-1}\left(p+\sigma_{i}\right)+c \prod_{i=1}^{l-1}\left(p+\xi_{i}\right) .
$$

If we let

$$
h_{0} p \prod_{i=1}^{l-1}\left(p+\sigma_{i}\right)=\sum_{i=0}^{l-1} h_{i}^{\prime} p^{l-1} \quad \text { and } \quad c \prod_{i=1}^{l-1}\left(p+\xi_{i}\right)=\sum_{i=1}^{l} h_{i}^{\prime \prime} p^{l-i},
$$

then the $h_{i}^{\prime}$ and $h_{i}^{\prime \prime}$ are all positive; and equating coefficients of like powers of $p$ in (2.13) gives

$$
h_{0}=h_{0}^{\prime}, \quad h_{i}=h_{i}^{\prime}+h_{i}^{\prime \prime} \quad(i=1,2, \cdots, l-1), \quad h_{l}=h_{l}^{\prime \prime} .
$$

Since $0 \leq g_{i} \leq h_{i}$ in (2.10), and in view of (2.14), there exists for each $i(i=1,2, \cdots$, $l-1)$ at least one pair $g_{i}^{\prime}, g_{i}^{\prime \prime}$ such that

$$
g_{i}=g_{i}^{\prime}+g_{i}^{\prime \prime}, \quad 0 \leq g_{i}^{\prime} \leq h_{i}^{\prime}, \quad 0 \leq g_{i}^{\prime \prime} \leq h_{i}^{\prime \prime} .
$$

Take $g_{0}^{\prime}=g_{0}$ and $g_{l}^{\prime \prime}=g_{l}$.

Now consider the functions

$$
\begin{gathered}
R_{1}(p)=\sum_{i=0}^{l-1} g_{i}^{\prime} p^{l-i} / h_{0} p \prod_{i=1}^{l-1}\left(p+\sigma_{i}\right)=\sum_{i=0}^{l-1} g_{i}^{\prime} p^{l-1-i} / h_{0} \prod_{i=1}^{l-1}\left(p+\sigma_{i}\right) \\
R_{2}(p)=\sum_{i=1}^{l} g_{i}^{\prime \prime} p^{l-1} / c \prod_{i=1}^{l-1}\left(p+\xi_{i}\right) .
\end{gathered}
$$

These are R-functions of degree $l-1$ at most. Hence by the hypothesis of induction there exist two 3 T.N. $\Gamma_{1}$ and $\Gamma_{2}$ whose transfer functions are $R_{1}$ and $R_{2}$ respectively, and whose $Y_{22}$ 's are

$$
Y_{22}^{(1)}=h_{0} \prod_{i=1}^{l-1}\left(p+\sigma_{i}\right) / \lambda_{1} S_{1} \quad \text { and } \quad Y_{22}^{(2)}=c \prod_{i=1}^{l-1}\left(p+\xi_{i}\right) / \lambda_{2} S_{2}
$$

respectively. Here $S_{1}$ and $S_{2}$ are polynomials of degree $l-2$ while $\lambda_{1}$ and $\lambda_{2}$ are arbitrary positive impedance-level constants whose values do not affect the transfer functions. We shall presently fix $\lambda_{1}$ and $\lambda_{2}$.

Now in view of (2.12) we can choose $\beta_{i}(i=1,2, \cdots, l-1)$ such that

$$
\xi_{1}<\beta_{1}<\sigma_{1}<\xi_{2}<\beta_{2}<\sigma_{2}<\cdots<\xi_{l-1}<\beta_{l-1}<\sigma_{l-1} .
$$

Hence

$$
Y_{22}^{(1)}=h_{0} p \prod_{i=1}^{l-1}\left(p+\sigma_{i}\right) /\left(p+\beta_{i}\right), \quad Y_{22}^{(2)}=c \prod_{i=1}^{l-1}\left(p+\xi_{i}\right) /\left(p+\beta_{i}\right)
$$

are both RC-admittances. We are going to modify the networks $\Gamma_{1}$ and $\Gamma_{2}$ so that their transfer functions remain unchanged but their $Y_{22}$ 's become $Y_{22}^{(1)}$ and $y_{22}^{(2)}$ respectively. 
In Fig. 2 consider the networks $\Gamma_{1}^{\prime}$ and $\Gamma_{2}^{\prime}$ where $Z_{1}$ and $Z_{2}$ are RC-impedances to be determined. Evidently the transfer functions of $\Gamma_{1}^{\prime}$ and $\Gamma_{2}^{\prime}$ are the same as those of $\Gamma_{1}$

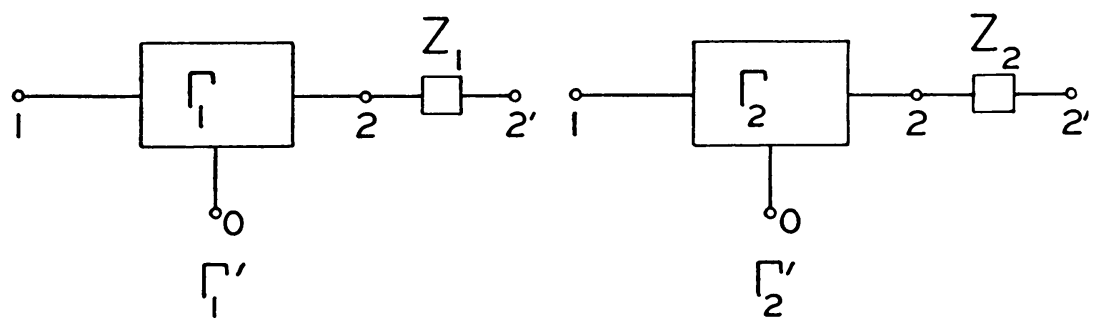

FIG. 2.

and $\Gamma_{2}$. However, the $Y_{22}$ 's of the new networks are $1 /\left(Z_{1}+1 / Y_{22}^{(1)}\right)$ and $1 /\left(Z_{2}+1 / Y_{22}^{(2)}\right)$ respectively. If these are to equal $Y_{22}^{(1)}$ and $Y_{22}^{(2)}$ respectively, then it is necessary and sufficient that $Z_{1}$ and $Z_{2}$ as given by

$$
Z_{1}=1 / \mathcal{Y}_{22}^{(1)}-1 / Y_{22}^{(1)}, \quad Z_{2}=1 / \mathcal{Y}_{22}^{(2)}-1 / Y_{22}^{(2)}
$$

be RC-impedances. Now in canonical form

$$
1 / \mathcal{Y}_{22}^{(1)}=B_{0} / p+\sum_{i=1}^{l-1} B_{i} /\left(p+\sigma_{i}\right), \quad 1 / \mathcal{Y}_{22}^{(2)}=C_{0}+\sum_{i=1}^{l-1} C_{i} /\left(p+\xi_{i}\right)
$$

with

$$
B_{i}>0, \quad C_{i}>0 \quad(i=0,1, \cdots, l-1)
$$

and

$$
1 / Y_{22}^{(1)}=\lambda_{1} \sum_{i=1}^{l-1} D_{i} /\left(p+\sigma_{i}\right), \quad 1 / Y_{22}^{(2)}=\lambda_{2} \sum_{i=1}^{l-1} E_{i} /\left(p+\xi_{i}\right)
$$

with

$$
D_{i} \geq 0, \quad E_{i} \geq 0 \quad(i=1,2, \cdots, l-1)
$$

Hence

$$
Z_{1}=B_{0} / p+\sum_{i=1}^{l-1}\left(B_{i}-\lambda_{1} D_{i}\right) /\left(p+\sigma_{i}\right), \quad Z_{2}=C_{0}+\sum_{i=1}^{l-1}\left(C_{i}-\lambda_{2} E_{i}\right) /\left(p+\xi_{i}\right),
$$

and by taking $\lambda_{1}$ and $\lambda_{2}$ sufficiently small and positive, $Z_{1}$ and $Z_{2}$ will be RC-impedances. The $Y_{12}$ 's of the networks $\Gamma_{1}^{\prime}, \Gamma_{2}^{\prime}$ in view of (2.1), are, respectively,

$$
\begin{aligned}
& y_{12}^{(1)}=y_{22}^{(1)} R_{1}=\sum_{i=0}^{l-1} g_{i}^{\prime} p^{l-1-i} / \prod_{i=1}^{l-1}\left(p+\beta_{i}\right), \\
& y_{12}^{(2)}=y_{22}^{(2)} R_{2}=\sum_{i=1}^{l} g_{i}^{\prime \prime} p^{l-i} / \prod_{i=1}^{l-1}\left(p+\beta_{i}\right) .
\end{aligned}
$$

Now connect the networks $\Gamma_{1}^{\prime}$ and $\Gamma_{2}^{\prime}$ in parallel to form a new 3 T.N., $\Gamma$. Then [6 p. 146] the $Y_{12}$ and $Y_{22}$ of $\Gamma$ are given by $Y_{12}=y_{12}^{(1)}+y_{12}^{(2)}, Y_{22}=y_{22}^{(1)}+y_{22}^{(2)}$. 
Replacing the $y_{12}$ 's and $y_{22}$ 's by their expressions given above and using (2.13) and (2.15) we find that the transfer function $A(p)$ of $\Gamma$ is $(2.10)$ while

$$
Y_{22}=\sum_{i=0}^{l} h_{i} p^{l-i} / \prod_{i=1}^{l-1}\left(p+\beta_{i}\right) \text {. }
$$

This completes the induction*.

The preceding synthesis procedure may be modified to accommodate any given resistive source and load. In the general case, this may require a finite reduction in the value of the maximum realizable $K$. However, if the source is zero or the load is infinite, all $K$ except possibly $K_{0}$ may be realized.

As an illustration, we outline the synthesis procedure for the case of a resistive load, which we may take as $1 \mathrm{ohm}$ without loss of generality. If $K<K_{0}$ we may suppose the R-function in (2.10) which corresponds to the transfer function is such that $0 \leq$ $g_{i}<h_{i}$. The procedure is now started by choosing $\tau_{i}(i=1,2, \cdots, l)$ such that $0<$ $\tau_{1}<\gamma_{1}<\tau_{2}<\cdots<\tau_{l}<\gamma_{l}$. Then as before we have

$$
h_{0} \prod_{i=1}^{l}\left(p+\gamma_{i}\right)=h_{0} \prod_{i=1}^{l}\left(p+\tau_{i}\right)+c^{\prime} \prod_{i=1}^{l-1}\left(p+\chi_{i}\right), \quad c^{\prime}>0,
$$

where $\tau_{1}<\chi_{1}<\tau_{2}<\chi_{2}<\cdots<\chi_{l-1}<\tau_{l}$. The original synthesis procedure is now applied to

$$
\sum_{i=0}^{l} g_{i} p^{l-i} / h_{0} \prod_{i=1}^{l}\left(p+\tau_{i}\right)
$$

to give a 3 T.N. $\Gamma$ whose $Y_{22}$ is

$$
\mu \prod_{i=1}^{l}\left(p+\tau_{i}\right) / \prod_{i=1}^{l-1}\left(p+\chi_{i}\right), \quad \mu>0 .
$$

If the $\tau_{i}$ are taken sufficiently close to the $\gamma_{i}$ this will always be possible. The network consisting of $\Gamma$ working into the load then realizes the original transfer function.

We conclude this section with the observation that theorems analogous to Theorem 1 exist for 3 T.N. having only resistance and (self) inductance or capacitance and (self) inductance. As shown in $[4$, Sec. 7] these are obtained from Theorem 1 by replacing $p$ by $1 / p$ in the RL case and by replacing $p$ by $p^{2}$ in the LC case.

3. An illustrative example. As an illustration of the foregoing theory and synthesis procedure consider the function $A(p)=K N / D=K\left(p^{2}-p+9\right) /(p+1)(p+14)$. First let us determine for what range of $K, A(p)$ is the transfer function of a 3 T.N. Since $m=n=2, a_{2}=9, b_{2}=14$, Theorem 1 tells us that $0<K<K_{0}$ where $K_{0}=$ $\min \left[K_{d}, 14 / 9,1\right]$. To calculate $K_{d}$ it is best to proceed indirectly by first eliminating $\kappa$ between the equations $D-{ }_{\kappa} N=0$ and $d / d p\left(D-{ }_{\kappa} N\right)=0$. This gives $16 p^{2}+$ $10 p-149=0$ whose roots $p_{1}=-3.380, p_{2}=2.755$ are the multiple roots of $D-$ $\kappa N=0$ which occur for all variations of $\kappa$. Only the latter of these is positive and corresponding to it we find $\kappa=D\left(p_{2}\right) / N\left(p_{2}\right)=4.546=K_{d}$. Hence $K_{0}=\min$ $[4.546,14 / 9,1]=1$.

*A variation of the above procedure may be obtained by modifying the decomposition as given in (2.13). Using either procedure an appropriate choice of (2.15) will frequently lead to simple network realizations. Still another modification of the synthesis procedure makes it possible to always choose $Z_{1}$ as a condenser and $Z_{2}$ as a resistor in Fig. 2 at each stage of the synthesis. 
Let us choose (as we may by Theorem 1) $K=K_{0}=1$. To apply the synthesis procedure, we must first write $A(p)$ as an $R$-function. Using the ideas of Appendix B (or by inspection) we find that the introduction of the common factor $(p+3)$ into $A(p)$ converts it into the R-function

$$
R=\left(p^{3}+2 p^{2}+6 p+27\right) /(p+1)(p+3)(p+14) .
$$

Following the procedure of $\S 2(b)$ choose $\sigma_{1}=2, \sigma_{2}=6$. Then (2.13) reads $p^{3}+18 p^{2}+$ $59 p+42=p\left(p^{2}+8 p+12\right)+\left(10 p^{2}+47 p+42\right)$, from which $\xi_{1}=6 / 5, \xi_{2}=7 / 2$. Now, for simplicity, in (2.15) let $2=2+0,6=0+6$; also choose $\beta_{1}=3 / 2, \beta_{2}=4$. Then we get

$R_{1}=\left(p^{3}+2 p^{2}\right) / p(p+2)(p+6)=p /(p+6), R_{2}=(6 p+27) / 10(p+6 / 5)(p+7 / 2)$, while according to $(2.16)$

and

$$
\mathcal{Y}_{22}^{(1)}=p(p+2)(p+6) /(p+3 / 2)(p+4)
$$

$$
\mathcal{Y}_{22}^{(2)}=10(p+6 / 5)(p+7 / 2) /(p+3 / 2)(p+4)
$$

respectively.

The transfer function $R_{1}$ being of first degree is realized by an $L$-network $\Gamma_{1}$ whose series and shunt arm impedances are $Z_{1 a}=\lambda_{1} / p$ and $Z_{1 b}=\lambda_{1} / 6$ respectively, and whose $Y_{22}^{(1)}=(p+6) / \lambda_{1}$. Use (2.17) to determine $Z_{1}$ taking $\lambda_{1}=3 / 8$ for simplicity. Then $Z_{1}=1 / 2 p+1 / 8(p+2)$. Finally form $\Gamma_{1}^{\prime}$ as in Fig. 2 .

As for $R_{2}$, it is of the second degree and hence the reduction procedure is repeated for it to obtain $R_{3}$ and $R_{4}$ of the first degree. We give the results, the notation being evident from what has preceded.

$$
\begin{aligned}
& R_{2}=\frac{6 p+27}{10(p+6 / 5)(p+7 / 2)}, \quad \begin{array}{ccccc}
\gamma_{1} & \xi_{1} & \beta_{1} & \sigma_{1} & \gamma_{2} \\
6 / 5, & 14 / 9, & 7 / 4, & 2, & 7 / 2
\end{array}, \\
& 10 p^{2}+47 p+42=10 p(p+2)+(27 p+42) ; \\
& R_{3}=0, \quad Y_{22}^{(3)}=\frac{10 p(p+2)}{(p+7 / 4)} ; \quad R_{4}=\frac{6 p+27}{27(p+14 / 9)}, \quad Y_{22}^{(4)}=\frac{27(p+14 / 9)}{(p+7 / 4)} .
\end{aligned}
$$

The transfer functions $R_{3}$ and $R_{4}$ are realized by L-networks $\Gamma_{3}, \Gamma_{4}$ whose series and shunt arm impedances are $Z_{3 a}=\infty, Z_{3 b}=(p+7 / 4) / 10 p(p+2) ; Z_{4 a}=\lambda_{4} /(6 p+27)$, $Z_{4 b}=\lambda_{4} /(21 p+15)$ respectively; and whose $Y_{22}$ 's are $Y_{22}^{(3)}=\mathcal{Y}_{22}^{*(3)}$ and $Y_{22}^{(4)}=$

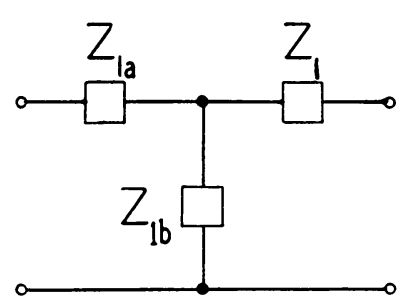

$\Gamma_{1}^{\prime}$

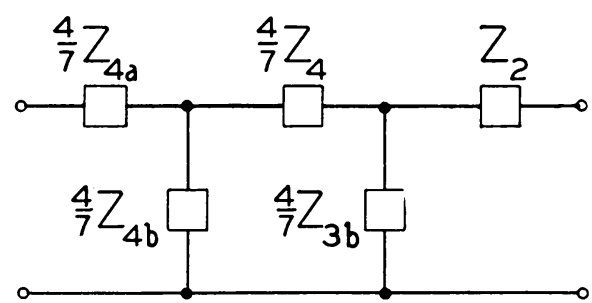

$\Gamma_{2}^{\prime}$

FIG. 3. 
$27(p+14 / 9) / \lambda_{4}$ respectively. Hence we need only determine a $Z_{4}$ using $(2.17)$ and taking $\lambda_{4}=7 / 36$. This gives $Z_{4}=1 / 27$. Now form $\Gamma_{4}^{\prime}$ as in Fig. 2 and denote by $\Gamma_{2}$ the parallel combination of $\Gamma_{3}$ and $\Gamma_{4}^{\prime}$. Introducing the impedance level factor $\lambda_{2}$ in $\Gamma_{2}$ the $Y_{22}$ of $\Gamma_{2}$ is given by $Y_{22}^{(2)}=10(p+6 / 5)(p+7 / 2) / \lambda_{2}(p+7 / 4)$. Again use (2.17) to determine $Z_{2}$ taking $\lambda_{2}=4 / 7$. Then $Z_{2}=1 / 10+4 / 175(p+6 / 5)$. Forming $\Gamma_{2}^{\prime}$ from $\Gamma_{2}$ and $Z_{2}$ as in Fig. 2, the required final network is the parallel combination of $\Gamma_{1}^{\prime}$ and $\Gamma_{2}^{\prime}$ as shown in Fig. 3.

4. The two terminal-pair network. Theorem 2. The necessary and sufficient conditions that a real rational function $A(p)$ given by (1.1) be the transfer function of an $R C-4 T . N$. are:

(i) The zeros of $D$ are distinct negative numbers.

(ii) $m \geq n$.

(iii) The number $K$ satisfies the inequalities $-K_{0}<K<K_{0}$, where $K_{0}$ is the least of the three quantities $\left|K_{d}\right|,\left|b_{m} / a_{n}\right|, 1$ if $m=n$ and of the first two quantities if $m>n$. If $K_{0} \neq\left|K_{d}\right|$ then $K$ may actually equal $\pm K_{0}$. Here $K_{d}$ is that real value of $\kappa$ of smallest absolute value (if it exists) for which the equation $D-{ }^{\circ} N=0$ has a positive multiple root.

Proof (a) Necessity. For (i) and (ii) see the remarks in Sec. 2. To prove (iii) consider the 4 T.N. of Fig. 4 on a nodal basis taking the input terminals as nodes 0 and 1, the

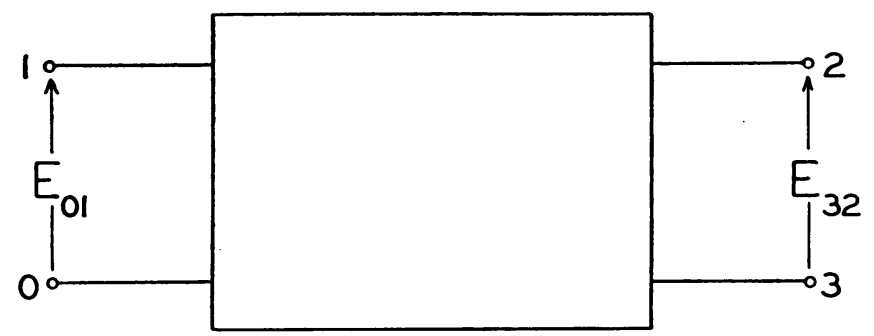

FIG. 4.

output terminals as nodes 2 and 3 , and choosing the remaining nodes as in the 3 T.N. case. Let $E_{01}$ and $E_{32}$ be the input and output voltages, respectively. Then in the notation of Sec. 2 we have $A(p)=E_{32} / E_{01}=\left(E_{2}-E_{3}\right) / E_{1}$. It follows from (2.2) that

$$
A(p)=\left(\Delta_{1}-\Delta_{3}\right) / \Delta_{2}
$$

with $\Delta_{1}$ and $\Delta_{2}$ as in (2.4), (2.5) and

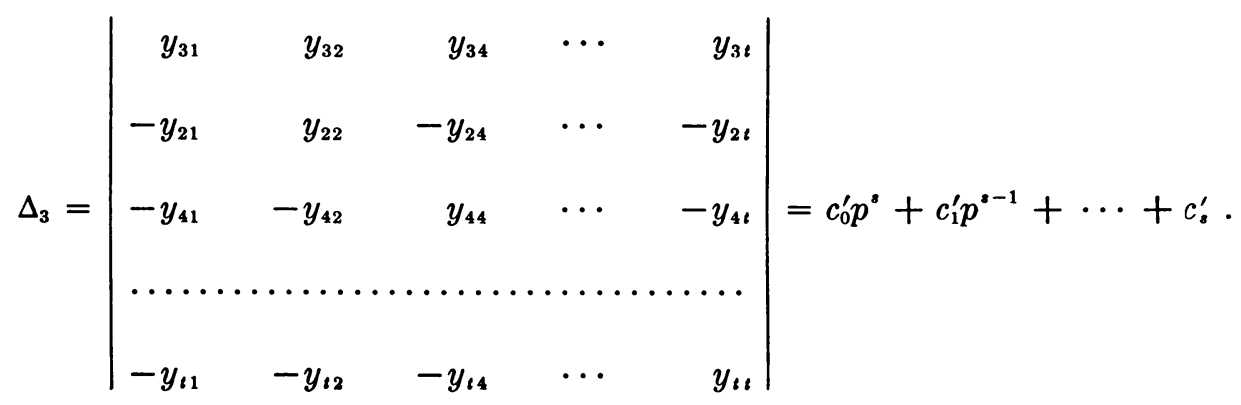


Since $\Delta_{3}$ is of the same form as $\Delta_{1}$ we have

$$
0 \leq c_{i}^{\prime} \leq d_{i} \quad(j=0,1, \cdots, s)
$$

in addition to (2.6).

If we equate the two expressions for $1 \pm A(p)$ obtained from (1.1) and (4.1), we have

$$
(D \pm K N) / D=\left[\Delta_{2} \pm\left(\Delta_{1}-\Delta_{3}\right)\right] / \Delta_{2} .
$$

Since the fraction on the left is evidently in reduced form, and in view of (2.6) and (4.2), it follows that $|K| \leq 1$ if $m=n$ and that $D \pm K N=0$ has no positive real roots. This in turn implies that $|K| \leq\left|b_{m} / a_{n}\right|$ if $a_{n} \neq 0$, and that $|K|<\left|K_{d}\right|$ for $K_{d}$ as defined in Theorem 2. This last fact follows as in the 3 T.N. case; for the "interval property" of $K$ holds for a 4 T.N. with $0<\left|K^{*}\right|<|K|$ in (2.9).

(b) Sufficiency. Let $A(p)$ satisfy the conditions of Theorem 2. Then proceeding as in Appendix B we find that both $D+K N$ and $D-K N$ have leading coefficients positive and have no positive real zeros. Thus there exists a polynomial $P$ having distinct negative real zeros such that $P(D+K N)$ and $P(D-K N)$ have non-negative coefficients. We may suppose $P$ and $D$ relatively prime. If

$$
P D=\sum_{i=0}^{l} h_{i} p^{l-i} \quad \text { and } \quad K P N=\sum_{i=0}^{l} g_{i} p^{l-i},
$$

it therefore follows that $\left|g_{i}\right| \leq h_{i}(i=0,1, \cdots, l)$. Write $A(p)=K P N / P D=$ $\left(N_{1}-N_{2}\right) / P D$, where $N_{1}$ consists of those terms of $K P N$ having positive coefficients while $-N_{2}$ consists of those terms of $K P N$ having negative coefficients. Then $N_{1} / P D$ and $N_{2} / P D$ are R-functions. Hence by the results of Sec. 2 there exist two 3 T.N., $\Gamma_{1}$ and $\Gamma_{2}$ whose ground, input and output terminals are $0,1,2$ and $0,1,2^{\prime}$ respectively, and whose transfer functions are $A_{1}=N_{1} / P D$ and $A_{2}=N_{2} / P D$ respectively. Now form the 4 T.N. shown in Fig. 5 whose input terminals are 0 and 1 and whose output

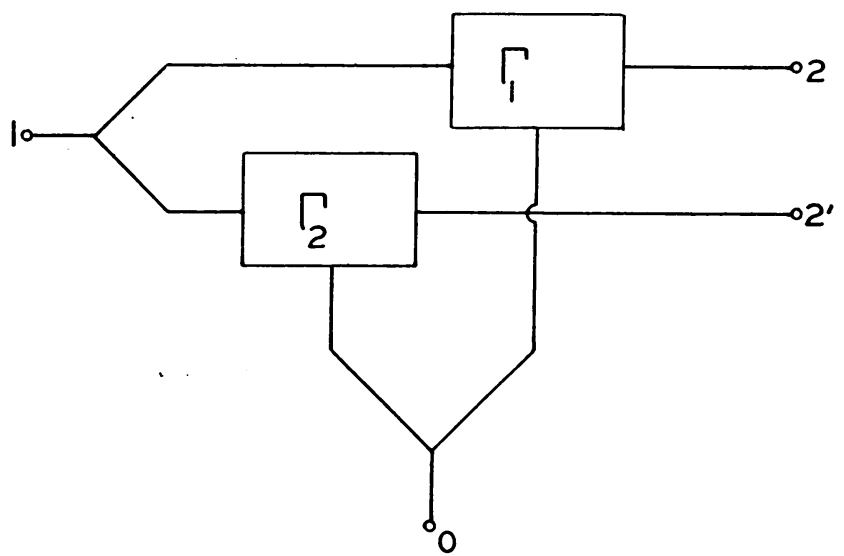

Fig. 5.

terminals are 2 and $2^{\prime}$. The transfer function of this network is evidently $A_{1}-A_{2}=A$. Remarks similar to those of Sec. 2 may also be made here concerning source and load, and the RL and LC networks. 


\section{Appendix A}

Consider the determinant

$$
H=\left|\begin{array}{ccccc}
a_{11} & a_{12} & a_{13} & \ldots & a_{1 n} \\
-a_{21} & a_{22} & -a_{23} & \ldots & -a_{2 n} \\
-a_{31} & -a_{32} & a_{33} & \ldots & -a_{3 n} \\
\ldots \ldots \ldots \ldots \ldots \ldots \ldots & \ldots \ldots \ldots \ldots \ldots \\
-a_{n 1} & -a_{n 2} & -a_{n 3} & \ldots & a_{n n}
\end{array}\right|,
$$

where $a_{i i}=\sum_{j=0, i \nVdash j}^{n} a_{i j}(i=2,3, \cdots, n)$ and all the $a_{i j}(i \neq j)$ and $a_{11}$ are independent variables. We shall prove that $H$ is a polynomial (multilinear form) in the $a_{i j}(i \neq j$ ) and $a_{11}$ with non-negative coefficients.

This is evidently true for $n=1,2$. Suppose it is true for determinants of the form $H$ of order less than $n(n \geq 3)$. We may write $H=H_{1}+H_{2}$ where $H_{1}$ contains those terms which are independent of $a_{2 n}(i=1,2, \cdots, n-1)$ and $H_{2}$ those terms which are linear in each $a_{i n}(i=1,2, \cdots, n-1)$ separately. In $H$, if we let $a_{i n}=$ $0(i=1,2, \cdots, n-1)$ we find

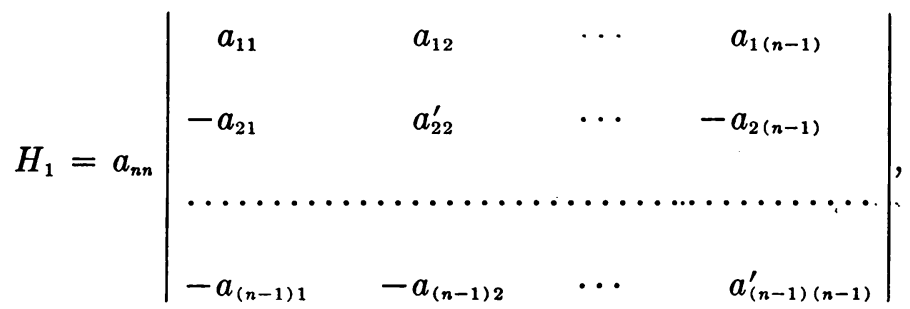

where $a_{i i}^{\prime}=a_{i i}-a_{i n}(i=2,3, \cdots, n-1)$. Hence applying the hypothesis of induction we conclude that the terms of $H_{1}$ have positive coefficients.

As for $\mathrm{H}_{2}$ each of its terms is contained in at least one of the polynomials $a_{i n} \partial H / \partial a_{i n}$ $(i=1,2, \cdots, n-1)$. Now

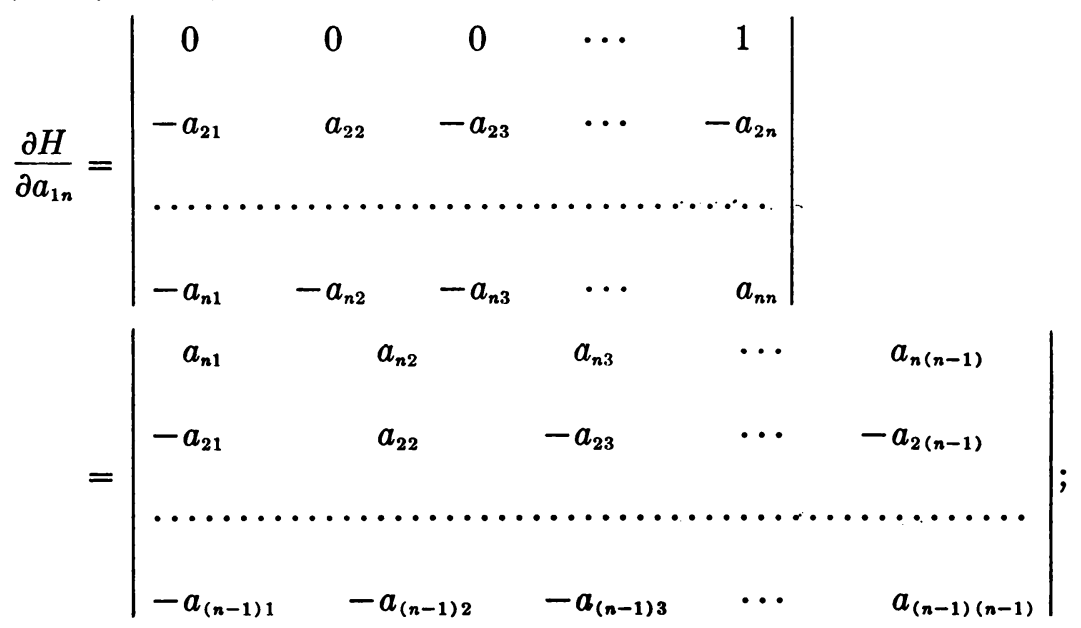


and for $i=2,3, \cdots, n-1$,

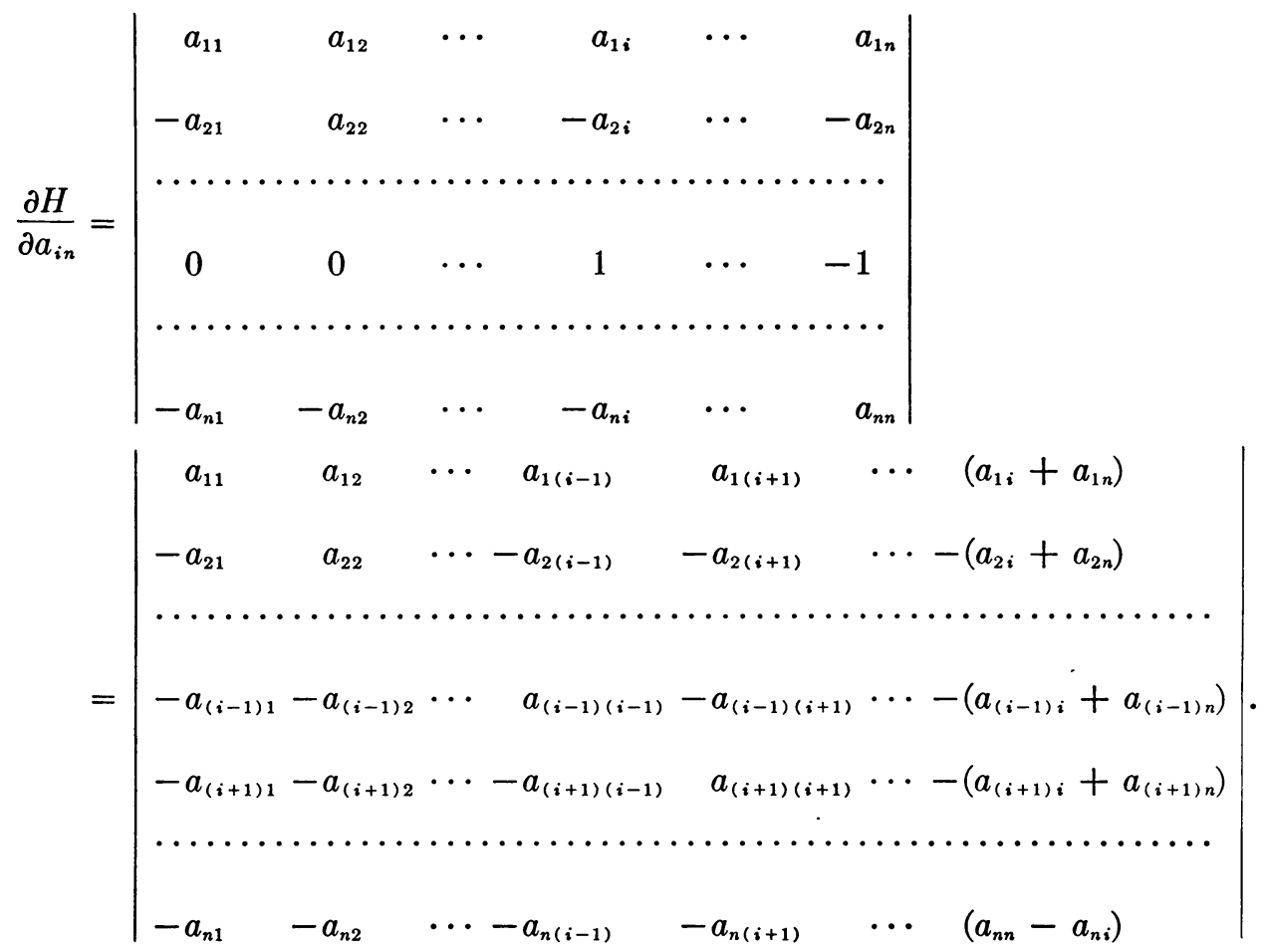

Replacing $a_{i 0}+a_{i n}(i=2,3, \cdots, n-1)$ by new variables $a_{i 0}^{\prime}$ in the first case, and $a_{i i}+a_{i n}(j=1,2, \cdots, n-1)$ by new variables $a_{i n}^{\prime}$ in the second case, we see that the final determinant in each case is reduced to a determinant of the same form as $H$ but of order $n-1$. Hence the terms of $H_{2}$ also have positive coefficients and the above italicized statement about $H$ is proved.

Now make the following specialization: $n=t-1, a_{i 1}=y_{(i+1) 1}(i=1,2, \cdots, t-1)$, $a_{i j}=y_{(i+1)(j+1)}(i=1,2, \cdots, t-1 ; j=2,3, \cdots, t-1 ; i \neq j), a_{i 0}=y_{(i+1) 2}+$ $y_{(i+1) 0}(i=1,2, \cdots, t-1)$. Then $H$ becomes $\Delta_{1}$ and we find that $\Delta_{1}$ is a polynomial in the $y_{i j}(i \neq j)$ with non-negative coefficients. This proves the $c_{i} \geq 0(i=0,1, \cdots, s)$ in (2.4).

In $\Delta_{1}$ replace the elements of the first column by $y_{20},-y_{30}, \cdots,-y_{t 0}$ respectively, to form $\Delta_{1}^{\prime}$. Then by symmetry $\Delta_{1}^{\prime}$ is also a polynomial in the $y_{i j}(i \neq j)$ with nonnegative coefficients. Now in $\Delta_{2}$ of $(2.5)$ add the sum of columns $2,3, \cdots, t-1$ to column 1. We see that $\Delta_{2}=\Delta_{1}+\Delta_{1}^{\prime}$. This proves that $d_{i} \geq c_{j}(j=0,1, \cdots, s)$.

\section{Appendix B}

Lemma*: Let $F(p)=\alpha p^{a}+\cdots, \alpha>0$ be a real polynomial having no positive real zeros. Then there exists a polynomial $P=\Pi\left(p+\delta_{i}\right)$ with the $\delta_{i}>0$ and distinct, such that $P F$ is a polynomial with non-negative coefficients.

*For a more general result which includes this one see [7]. We include the following simple constructive proof so as to keep the paper self-contained with regard to the synthesis procedure. 
The real irreducible factors of $F$ are either of the form $p+\tau$ with $\tau \geq 0$ or $p^{2}-2 \rho \cos \varphi p+\rho^{2}$ with $\rho>0,0<\varphi<2 \pi$. It clearly suffices to prove the lemma for $F$ equal to this latter quadratic polynomial with $0<\cos \varphi<1$. Replacing $p$ by $\rho p$ we may without loss of generality suppose that $\rho=1$.

Let $P_{1}=(p+1)^{b}$ where $b$ is an integer such that

$$
b>2 \cos \varphi /(1-\cos \varphi) .
$$

We have

$$
P_{1} F=\sum_{i=0}^{b+2} \theta_{i} p^{i}
$$

where

$$
\theta_{i}=\left(\begin{array}{c}
b \\
i
\end{array}\right)-2 \cos \varphi\left(\begin{array}{c}
b \\
i-1
\end{array}\right)+\left(\begin{array}{c}
b \\
i-2
\end{array}\right) \quad(i=0,1, \cdots, b+2)
$$

and $\left(\begin{array}{l}b \\ i\end{array}\right)$ is the binomal coefficient, with $\left(\begin{array}{l}b \\ i\end{array}\right)$ defined as zero if $i>b$ or $i<0$.

Now $\theta_{0}=\theta_{b+2}=1>0$; and $\theta_{1}=\theta_{b+1}=b-2 \cos \varphi>0$ by (B.1). If $2 \leq i \leq b$,

$$
\theta_{i}=\left(\begin{array}{c}
b \\
i-1
\end{array}\right)\left[\frac{(b-i+1)(b-i+2)+i(i-1)}{i(b-i+2)}-2 \cos \varphi\right] .
$$

We have $i(b-i+2) \leq(i+b-i+2)^{2} / 4=(b+2)^{2} / 4$; and $(b-i+1)(b-i+2)+$ $i(i-1)=\left\{b(b+2)+[b-2(i-1)]^{2}\right\} / 2 \geq b(b+2) / 2$. Hence in (B.2), the bracket satisfies the inequality, [ $] \geq 2 b /(b+2)-2 \cos \varphi>0$ by (B.1). Since the coefficients $\theta_{i}$ of $P_{1} F$ are all positive we may by continuity considerations form $P_{1}^{\prime}=$ $\prod_{i=1}^{b}\left(p+\delta_{i}\right)$ with the $\delta_{i}$ sufficiently close to 1 and distinct, such that $P_{1}^{\prime} F$ has positive coefficients. This proves the lemma. We note that the method used in the above proof may not necessarily lead to the simplest polynomial $P$.

Now let $A(p)$ satisfy the conditions of Theorem 1 . Then evidently $K N$ is a polynomial such as $F$ in the lemma. But $D-K N$ is also such a polynomial! To prove this we first show that $D-K N$ has no positive real zeros. Consider the zeros $\zeta_{i}(\kappa)$ of $D-$ $\kappa N=0$ where $\kappa$ is a non-negative, real parameter. When $\kappa=0$ these zeros are the zeros of $D$ and hence are negative real and distinct. Then for small positive $\kappa$ the $\zeta_{i}$ will be negative real and distinct. As $\kappa$ increases positively, positive real $\zeta_{i}$ may arise only if one of the following has occurred: (a) a $\zeta_{i}$ has gone through zero, (b) a $\zeta_{i}$ has gone through $\infty,(\mathrm{c})$ two or more complex $\zeta_{i}$ have become equal on the positive real axis. In case (a) we must have $\kappa>b_{m} / a_{n}\left(a_{n} \neq 0\right)$; in case (b) $\kappa>1$ if $m=n$; in case (c) $\kappa \geq K_{d}$. Hence, by (iv) in Theorem 1 , if $\kappa=K, D-K N$ has no positive real zeros.

We must still show that the leading coefficient of $D-K N$ is positive. This is evident unless $m=n, K=K_{0}=1$. In this case, we have $D-N=\sum_{i=0}^{r}\left(b_{m-i}-a_{m-i}\right) p^{i}$, $b_{m-r}-a_{m-r} \neq 0, r<m$. Suppose the leading coefficient $b_{m-r}-a_{m-r}<0$. Consider the polynomial $Q_{1}=\sum_{i=0}^{r} e_{m-i} p^{i}$, where $e_{m-i}=b_{m-i}-a_{m-i}$ if $a_{m-i}<0$ and $e_{m-i}=$ $b_{m-i}-\left(1-\epsilon_{1}\right) a_{m-i}, \epsilon_{1}>0$ if $a_{m-i} \geq 0$. For $\epsilon_{1}$ sufficiently small the leading coefficient of $Q_{1}$ will be negative so that there exists a $p_{0}>0$ such that $Q_{1}\left(p_{0}\right)<0$. Now 


$$
\begin{aligned}
D\left(p_{0}\right)-(1-\epsilon) N\left(p_{0}\right)=\epsilon \sum_{i=r+1}^{m} a_{m-i} p_{0}^{i} & +\sum_{i=0}^{r}\left[b_{m-i}-(1-\epsilon) a_{m-i}\right] p_{0}^{i} \\
& <\epsilon \sum_{i=r+1}^{m} a_{m-i} p_{0}^{i}+Q_{1}\left(p_{0}\right) \text { for } 0<\epsilon<\epsilon_{1} .
\end{aligned}
$$

Hence for $\epsilon$ sufficiently small, say $\epsilon=\epsilon_{2}<\epsilon_{1}, D\left(p_{0}\right)-\left(1-\epsilon_{2}\right) N\left(p_{0}\right)<0$ while $D(\infty)-\left(1-\epsilon_{2}\right) N(\infty)>0$. Thus $D(p)-\left(1-\epsilon_{2}\right) N(p)=0$ has a positive real root, which contradicts what has been proved above about $D-\kappa N=0$ for $\kappa<K_{0}$. Thus by the lemma, there exist two polynomials $P_{1}$ and $P_{2}$ such that $K P_{1} N$ and $P_{2}(D-K N)$ have non-negative coefficients. Then $P_{1} P_{2}$ will simultaneously convert $K N$ and $D-K N$ into polynomials with non-negative coefficients. We may suppose $P_{1}, P_{2}$ and $D$ are relatively prime in pairs. Now let

$$
A(p)=K N / D=K P_{1} P_{2} N / P_{1} P_{2} D=\sum_{i=0}^{l} g_{i} p^{l-i} / \sum_{i=0}^{l} h_{i} p^{l-i}
$$

Then the zeros of the denominator of the last fraction are negative real and distinct, and by considering $K P_{1} P_{2} N$ and $P_{1} P_{2}(D-K N)$ it follows that $0 \leq g_{i} \leq h_{i}(i=0,1, \cdots, l)$. Hence the last fraction is an R-function.

We remark that if $K_{0}=1$ or $b_{m} / a_{n}$ and if $R_{0}$ is an R-function corresponding to $K_{0}$, then $K R_{0} / K_{0}$ corresponds to any $K$ in the range $0<K \leq K_{0}$. However, if $K_{0}=K_{d}$, no such $\mathrm{R}$-function $R_{0}$ exists and the degrees of the R-functions corresponding to $K$ in the range $0<K<K_{d}$ must increase indefinitely as $K \rightarrow K_{d}$.

\section{References}

1. H. W. Bode, Network analysis and feedback amplifier design, D. Van Nostrand, Inc., New York, 1945, pp. 11-12.

2. J. L. Bower and P. F. Ordung, The synthesis of resistor-capacitor networks, Proc. I. R. E. 38, 263-269 (1950).

3. L. E. Dickson, First course in the theory of equations, John Wiley and Sons, New York (1922), pp. 146, 152.

4. A. Fialkow and I. Gerst, The transfer function of an RC ladder network, J. Math. Physics, 30, 49-72, (1951).

5. E. A. Guillemin, Synthesis of RC-networks, J. Math. Physics, 28, 22-42 (1949).

6. E. A. Guillemin, Communication networks, Vol. 2, John Wiley and Sons, New York, 1935.

7. G. Pólya and G. Szegö, Aufgaben und Lehrsätze aus der Analysis, vol. 2, Dover, New York, 1945, p. 73, ex. 190 . 